Atıf için / For Citation: G. BOZ, M. OKUTAN, B. DEMIR, "Nazofarenks Kanseri Radyoterapi Tedavisinde Hibrit Tedavi Planının TLD ile Dozimetrik Doğrulanması", Süleyman Demirel Üniversitesi Fen Edebiyat Fakültesi Fen Dergisi, 14(2), 395-401, 2019.

\title{
Nazofarenks Kanseri Radyoterapi Tedavisinde Hibrit Tedavi Planının TLD ile Dozimetrik Doğrulanması
}

\author{
Gamze BOZ ${ }^{1}$, Murat OKUTAN ${ }^{* 1}$, Bayram DEMIR ${ }^{2}$ \\ ${ }^{1}$ İstanbul Üniversitesi, Onkoloji Enstitüsü, Sağlık Fiziği Bölümü, 34104, İstanbul, Türkiye \\ ${ }^{2}$ Ístanbul Üniversitesi, Fen Fakültesi, Fizik Bölümü, 34126, İstanbul, Türkiye \\ *yazışılan yazar e-posta: mokutan@istanbul.edu.tr
}

(Alınış / Received: 11.10.2019, Kabul / Accepted: 14.11.2019, Yayımlanma / Published: 30.11.2019)

Özet: Nazofarenks kanserinde geleneksel tedavi planlama, günümüzde yerini optimizasyon tekniklerinin kullanıldığı tersten planlamaya Yoğunluk Ayarlı Radyoterapi - Yoğunluk Ayarlı Arc Terapi (IMRT-IMAT) ve ikisinin birlikte kullanıldığ 1 Hibrit tedavi planlamalara bırakmıştır. Özellikle IMAT veya IMRT tedavi planlarında düşürülemeyen kritik organ dozları veya iyi kapsanamayan PTV hacimleri için Hibrit planlara ihtiyaç duyulmaktadır. Bu karmaşık hesaplamaları yapan Tedavi Planlama Sistemlerinin (TPS) doz hesaplarının dozimetrik yöntemlerle doğrulanması gerekmektedir. Bu çalışmada Termolüminesans dozimetre (TLD) yöntemi ile TPS doğruluğu test edilmiştir. Alderson ${ }^{\circledR}$ Rando Fantom'un nazofarenks kanseri tedavi planlaması için tedavi şartlarında bilgisayarlı tomografi görüntüleri çekilmiştir. Planlanan Hedef Hacim 70 Gy (PTV70) ve kritik organlar (sağ parotis, sol parotis, medulla ve beyin sapı) tespit edilirken standart bir nazofarenks tümörlü hastanın Bilgisayarlı tomografi kesitlerinden füzyon yapılarak yararlanılmışıır. IMRT, IMAT ve Hibrit tedavi planları Eclipse (version 8.9.17) TPS kullanılarak fantom üzerinde aynı doz reçetesi ile oluşturulmuştur. Alderson ${ }^{\circledR}$ Rando Fantomda sağ ve sol parotise, medulla spinalise ve beyin sapına denk gelecek şekilde yerleştirilen TLD'lerin okuma değerleri toplam doza dönüştürülerek tedavi planlama bilgisayarındaki doz değerleriyle karşılaştırılmıştır. Eclipse TPS ile TLD ortalama okuma değerleri arasında en fazla fark IMRT planında Sol Parotiste \% 3.5, IMAT planında Beyin Sapında \% 2.78, Hibrit planda Beyin Sapında \% 3.16 olarak bulunmuştur. Bu çalışma ile Nazofarenks için yapılan Hibrit tedavi planının TPS de doğruluğunun \% 4 ten az olduğunu dozimetrik olarak tespit ettik.

Anahtar kelimeler: Nazofarenks, Eclips TPS, Hibrit plan, TLD

\section{Dosimetric Verification of Hybrid Treatment Plan by TLD in the Treatment of Nasopharyngeal Cancer Radiotherapy}

\begin{abstract}
Traditional treatment planning in nasopharyngeal cancer is now replaced by reverse planning using optimization techniques Intensity Modulated Radiotherapy-Intensity Modulated Arc Therapy (IMRT-IMAT) and Hybrid therapy planning using both. Hybrid plans are needed especially for critical organ doses that cannot be reduced in IMAT or IMRT treatment plans, or for PTV volumes that are not well covered. The dose calculations of the Treatment Planning Systems (TPS) that make these complex calculations need to be verified by dosimetric methods. In this study, TPS accuracy was tested by Thermoluminescence dosimeter (TLD). Computed tomography images of the Alderson ${ }^{\circledR}$ Rando Phantom for treatment planning in nasopharyngeal cancer were scanned. Planned Target Volume 70 Gy (PTV70) and critical organs (right parotid, left parotid, medulla and brain stem) were detected while computed tomography sections of a standard nasopharyngeal tumor patient were utilized by fusion. IMRT, IMAT and Hybrid
\end{abstract}


treatment plans were created with the same dose prescription on the phantom using Eclipse (version 8.9.17) TPS. In Alderson ${ }^{\circledR}$ Rando Phantom, TLDs placed in the right and left parotid, medulla spinalis and brain stem were converted to cumulative doses and compared with the dose values on the treatment planning computer. The highest difference between Eclipse TPS and TLD mean reading values was found to be $3.5 \%$ in the left parotid IMRT plan, $2.78 \%$ in the brain stem in the IMAT plan and $3.16 \%$ in the brain stem in the hybrid plan. In this study, we determined dosimetric accuracy of the Hybrid treatment plan for nasopharynx is less than 4 $\%$ in TPS.

Key words: Nasopharynx, Eclipse TPS, Hybrid plan, TLD

\section{Giriș}

Nazofarenks kanseri (NFK) komşu bölgelere erken dönemde doğrudan yayılım özelliği gösterir, çevre yapılarda kemik hasarı fazladır (\%25-35) ve parafarengeal alana yayılımı sıklıkla gözlenir (\%65-90). Aynı zamanda lenfatik ağı zengin olduğu için erken dönemde lenfatik tutulum yapar. Tedavi için başvuruda hastaların (\%65-80) önemli bölümünde lenf nodlarında tutulum görülür (1-3). Radyoterapi (RT) tedavisi NFK için birincil tedavi yöntemidir ve ileri evre NFK'nde kemoterapi ile birlikte uygulanabilir. NFK' lı hastalarda RT ile erken evrelerde başarı şansı \%80-95 oranında, ilerlemiş hastalıklar da \%40-70 oranlarındadır. Lokal ve bölgesel ilerlemiş tümörlerde eşzamanlı kemoterapi kullanımının sağkalımı arttırdığı gösterilmiştir (4,5). Geleneksel tedavi planlama, günümüzde yerini target ve kritik dokuların doz sınırlamalarının kullanıcı tarafından belirlendiği optimizasyon tekniklerinin kullanıldığı tersten planlamaya Yoğunluk Ayarlı Radyoterapi (IMRT) - Yoğunluk Ayarlı Arc Terapi (IMAT) bırakmıştır. IMRT düzgün olmayan akıya sahip alanların hastada istenilen doz dağılımını optimize etmek için kullanıldığı bir radyoterapi tekniğidir. IMAT tekniğinin kliniğe uygulanması için düzgün olmayan akı haritaları oluşturabilen bir tedavi planlama sistemi ve bu planlamanın tam olarak uygulanabilmesini sağlayan bir tedavi cihazı gerekmektedir $(6,7)$. IMAT 'nin eşdeğer veya daha iyi hedef kapsama alanı sunduğunu gösteren çok sayıda çalışma yapılmıştır ve çok daha düşük Monitor Unit (MU)'larla ve daha kısa tedavi süresi ile korunan risk altındaki organları (OAR'lar) büyük ölçüde iyileştirmektedir $(8,9,10)$. Fakat, literatürde IMAT ile IMRT arasındaki dozimetrik karşılaştırmalarda farklı sonuçlar vardır. Ning ve ark. (11), tek arklı ve çift arklı planlar için hedef doz homojenliği ve uygunluğunu 9 alanlı IMRT 'ye göre daha üstün olduğunu bulmuşken, 9 alanlı IMRT de parotis bezinin korunmasının ark planlara göre daha iyi olduğunu gözlemlemişlerdir. Literatürde IMRT ve IMAT'1 birleştiren hibrid teknik, IMRT ve IMAT ile karşılaştırılan doz dağılımını iyileştirmek için potansiyel bir fayda sağlamaktadır (12). Özellikle bu tedavi bölgesinde kritik organ ve Planlanan Hedef Hacim (PTV)'lerin iç içe bulunması, kolimatör yapısındaki Multi Leaf Kolimatör (MLC)'lerin tedavi planlama sistemine (TPS) doğru modellenmesi gerekliliği önem kazanmaktadır. Bu yüzden, bu hesaplamaları yapan TPS'lerin de doz hesaplarının dozimetrik yöntemlerle doğrulanması gerekmektedir. Biz de çalışmamızda; Nazofarenks tümörlerinde IMRT, IMAT ve hibrit tedavi teknikleri kullanılarak rando fantom üzerinde yapılan planlarda bu teknikler için kritik organlar ve hedef hacim dozlarının Termolimünesans Dozimetri (TLD) ile dozimetrik olarak TPS ile karşılaştırılmasını amaçladık. 


\section{Materyal ve Metot}

\subsection{Alderson ${ }^{\circledR}$ Rando Fantomun Hazırlanmast}

Çalışmamızda rando fantomun başından göğüs hizasına kadar olan 15 kesit kullanıldı. Kullandığımız rando fantomun, her bir kesiti $2,5 \mathrm{~cm}$ kalınlığında olup kullanılan fantomun toplam uzunluğu $37,5 \mathrm{~cm}$ 'dir. Fantomu oluşturan kesitlerin birbirinden ayrılmaması ve doz dağılımını etkileyecek yanlışlıklara (hava boşlukları, kayma vb.) yer vermemek amacıyla fantom sıkı bir şekilde bantlanarak kullanıldı.

\subsection{Alderson ${ }^{\circledR}$ Rando Fantomun Bilgisayarlı Tomografisi ve Konturlanması}

Alderson ${ }^{\circledR}$ Rando fantom Philips Brilliance Big Bore CT-simülatör cihazına supin olarak yatırıldı. Baş boyun maskesi yapıldı. $3 \mathrm{~mm}$ aralıklı kesitlerle görüntüleri alındı. BT görüntüleri bir hastanın çizilmiş tedavi ve kritik organ hacimlerinin fantoma füzyonu ile çizildi. Gross tümör hacmi (GTV), Klinik hedef hacmi (CTV), PTV'leri oluşturmak için tüm hacimlerde CTV'lere $3 \mathrm{~mm}$ 'lik bir marj eklendi. PTV'ler cilt yüzeyinden 3 mm'ye kadar kesildi. OAR'larda beyin sap1, omurilik, optik sinirler, optik çatlama, parotis bezleri, temporomandibular eklemler, mandibula, gözler, lensler, temporal loblar ve tanımlanmamış doku dahil edildi. Omurilik, PTV'nin üst sınırının en az $2 \mathrm{~cm}$ üzerinde başlayarak ve her BT diliminde PTV'nin alt sınırının en az $2 \mathrm{~cm}$ altına kadar devam ettirilerek konturlandı. Alınan görüntüler Eclipse TPS'ne aktarıldı.

\subsection{Tedavi Planının Hazırlanması}

120 yapraklı Millennium Multileaf Collimator (MLC) ile donatılmış Varian Trilogy linac'dan üretilen $6 \mathrm{MV}$ foton 1şınları ve tedavi planlaması için Eclipse 8.9 (Varian Medical Systems, Inc., Palo Alto, CA) tedavi planlama sistemi kullanıldı.

IMRT planı için, yedi farklı alan oluşturuldu. Gantri açıları $0^{\circ}, 52^{\circ}, 104^{\circ}, 156^{\circ}, 208^{\circ}$, $260^{\circ}, 312^{\circ}$ tedavi sliding window tekniği ile SIB (Simültene integre boost) şeklinde planlandı. Planlamaya hedef hacimlerin alması istenen doz değerleri (PTV70, PTV60, PTV54'ün reçete edilen dozun \% 95'ini kapsayacak şekilde plan normalizasyonuyla) ve kritik organların da (parotisler, beyin sapı, medulla vs.) aşmaması gereken tolerans değerleri girilerek doz reçeteleri oluşturuldu ve optimizasyon işlemi gerçekleştirildi.

IMAT planı için, IMRT doz reçetesine bağlı kalarak üç adet tam ark kullanılarak plan yapıldı. Birinci ark alanında $330^{\circ}$ ' lik kolimatör açısı seçilerek $179.9^{\circ}-180.1^{\circ}$, ikinci arc alanında $30^{\circ}$ lik kolimatör açısı seçilerek $180.1^{\circ}-179.9^{\circ}$, gantri açıları $179.9^{\circ}-180.1^{\circ}$ ve $90^{\circ}$ kolimatör ile üç tam arc kullanıldı.

Hibrit planı için, IMRT ve IMAT planlama parametreleri ve optimizasyon uygulamaları kombine edilerek, reçete edilen dozun yarısı IMRT planından, diğer yarısı ise IMAT planından verilerek Hibrit planı oluşturuldu.

\subsection{Alderson ${ }^{\circledR R a n d o}$ Fantoma TLD GR-200A Rodların Yerleştirilmesi}

Radyoterapide en çok kullanılan TL malzemelerden olan GR-200A rodları lityum florür kökenli olup; magnezyum, bakır ve fosfor ile aktive edilmiştir. Bu dozimetreler çip, rod ve toz şeklindedirler. Yüksek hassasiyete sahiptirler.Geniş bir doz aralığında $(0,5 \mu \mathrm{Gy}-$ 18 Gy) ölçüm yapabilirler. Düşük dozları ölçebildikleri için bu dozimetrelerle hastada 
uzak alan dışı doz ölçümleri, çevresel ve radyolojik dozimetri yapılabilir. GR-200 A termolüminesans rodları 1x1x6 mm boyutlarındadır.

Alderson ${ }^{\circledR}$ Rando fantomu 1şınlamadan önce fantomun kesitleri birbirinden ayrıld1. Alderson ${ }^{\circledR}$ Rando fantom üzerinde belirlenen 5. ve 6 . kesitlerde parotislere denk gelicek şekilde 2 adet TLD çubukları yerleştirildi. 6. ve 7. Kesitler arasında kalacak şekilde medullaya 1 adet TLD ve yine 5. ve 6. kesitler arasında beyin sapına denk gelecek şekilde 1 adet TLD yerleştirildi (Şekil 1). Daha sonra fantomun kesitleri tekrar birleştirilerek sabitlendi ve ışınlamaya hazır hale getirildi.

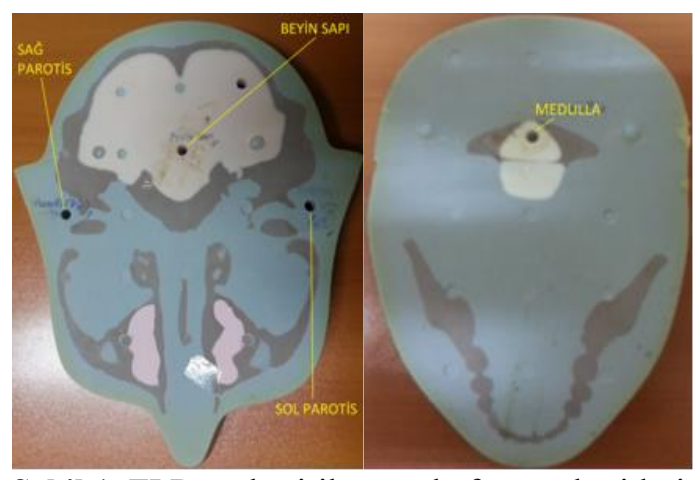

Şekil 1. TLD yerleştirilen rando fantom kesitleri.

\subsection{Alderson ${ }^{\circledR}$ Rando Fantomun ışınlanması}

Alderson ${ }^{\circledR R a n d o}$ Fantomun 1şınlanmasından önce lineer hızlandırıcıda da doz kalibrasyonu yapılmıştır. Kalibrasyonu yapılan TLD rodları standart sapmaları \%1 içinde olacak şekilde grupland. TLD ler Fantom üzerinde Sağ ve Sol parotis, medulla spinalis ve beyin sapına denk gelecek yerlere (Şekil 1) yerleştirilerek ışınlama yapıldı. Fantomun 1şınlamaları Varian marka Trilogy cihazında yapılmıştır. Işınlama; IMRT, IMAT ve Hibrit planları için iki fraksiyonluk doz verilerek üç kez tekrarlanarak yapıldı.

\subsection{TLD'lerin Kalibrasyonu}

Kalibrasyon 1şınlaması, Kobalt-60 cihazında verilen radyasyonu soğuran dozimetrenin TL yanıtını belirlemek için yapılmıştır. Kalibrasyon ışınlaması için GR-200 A rodları $10 x 10 \mathrm{~cm}$ 'lik alan içerisinde $6 \mathrm{~mm}$ çapında 1mm derinliğinde 80 delikten oluşan TLD' lerin olduğu pleksiglas tepsi içerisine yerleştirilmiştir. Kobalt (Co-60) cihazının tedavi masasına pleksiglas tepsi, RW-3 katı su fantomları ile $5 \mathrm{~cm}$ derinlikte olacak şekilde yerleştirilmiştir. Deney düzeneği Şekil 2 de verilmiştir.

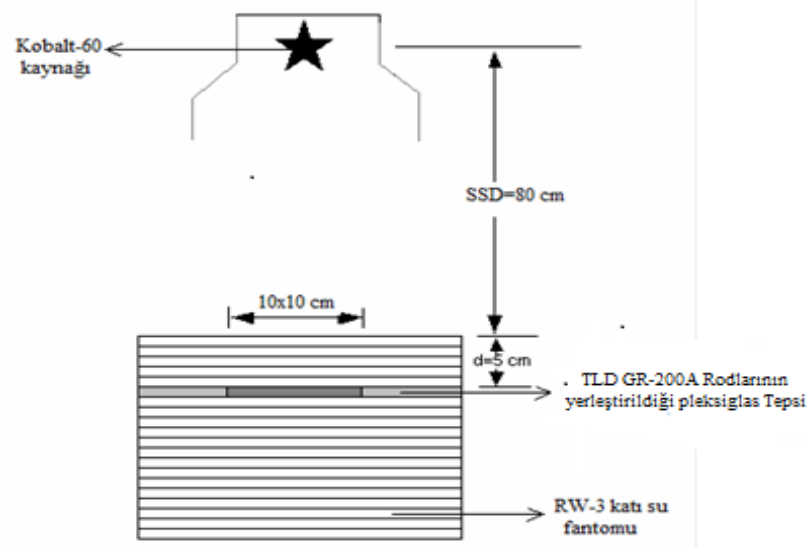

Şekil 2. TLD kalibrasyon düzeneği. 


\section{Bulgular}

Planların dozimetrik kalitesi CI ve HI, ICRU Rapor 83' e (6) göre hesaplanmıştır. Buna göre; IMRT planı için CI:1.045 IMAT planı için CI:1.026, IMRT planı için HI:0.082 ve IMAT planı için HI:0.07 bulunmuştur.

Alderson ${ }^{\circledR R}$ Rando Fantomda sağ ve sol parotise, medulla spinalise ve beyin sapına denk gelecek şekilde yerleştirilen TLD' lerin okuma değerleri toplam doza dönüştürülerek tedavi planlama bilgisayarındaki doz değerleriyle karşılaştırılmıştır (Tablo1). Eclipse TPS ile TLD ortalama okuma değerleri arasında en fazla fark IMRT planda Sol Parotiste \%3.5, IMAT planda Beyin Sapında \%2.78, Hibrit planda Beyin Sapında $\% 3.16$ olarak bulunmuştur.

Çalışmamızda IMRT ve IMAT teknikleri ve aynı doz reçeteleriyle oluşturulan planlarda 1şınlama süreleri sırasıyla; 15 dakika ve 4 dakika olarak bulunmuştur. MU değerleri ise sırasıyla 1224 MU ve 442 MU olarak hesaplanmıştır.

Tablo 1. IMRT,IMAT ve Hibrit planlar için kritik organ (Sağ parotis, Sol parotis, Medulla Spinalis ve Beyin Sap1) TLD okumaları (Gy) ve aynı noktadaki TPS okumaları arasındaki farklar (\%)

\begin{tabular}{ccccc}
\hline & Să̆ Parotis & Sol Parotis & Medulla Spinalis & Beyin Sap \\
IMRT, TLD (Gy) & $15.83 \pm 1.35$ & $14.33 \pm 0.81$ & $35.12 \pm 1.08$ & $41.58 \pm 1.27$ \\
TPS-Okuma (Gy) & 15.67 & 14.85 & 34.25 & 41.27 \\
Fark (\%) & 1.01 & 3.50 & 2.54 & 0.75 \\
IMAT, TLD (Gy) & $13.72 \pm 0.57$ & $12.54 \pm 0.49$ & $33.25 \pm 1.32$ & $38.04 \pm 1.302$ \\
TPS-Okuma (Gy) & 14.05 & 12.40 & 33.85 & 37.01 \\
Fark (\%) & 2.40 & 1.12 & 1.77 & 2.78 \\
HİBRİT,TLD (Gy) & $14.51 \pm 0.4$ & $13.62 \pm 0.49$ & $33.6 \pm 0.98$ & $38.6 \pm 1.05$ \\
TPS-Okuma (Gy) & 14.36 & 13.45 & 34.15 & 39.82 \\
Fark (\%) & 1.04 & 1.26 & 1.61 & 3.16 \\
\hline
\end{tabular}

\section{Sonuç ve Yorum}

Çalışmamızda IMRT ve IMAT teknikleri ile yapılan planlarda sırasıyla CI değerleri 1,045 ve 1,026 olarak bulunmuştur. PTV70' deki doz dağılımının homojenliğini karşılaştırmak amacıyla IMRT ve IMAT teknikleri ile yapılan planlarda sirasıyla HI değerleri 0,082 ve 0,07 olarak bulunmuştur. Yıng Sun ve ark. (13) yaptığı çalışmada bizim çalışmamızda olduğu gibi \%95 lik izodoza tanımlanan PTV70 hacmi için CI değerleri IMRT ve IMAT planları için sırasıyla 1,07 ve 1,09, HI değerlerini sırasıyla 0,54 ve 0,58 bulunmuşlardır.

Laurence E. Court ve ark. (14) Sinüs bölgesindeki tümörlerin Dinamik IMRT ile tedavisinde MC hesaplamaları ile TPS hesaplamalarını iyon odası ölçümleriyle karşılaştırdıkları çalışmada farkın $\% 5$ ten az olduğunu bildirmişlerdir.

Tao Han ve ark. (15) Baş boyun fantomunda IMRT ve IMAT planları için planlama algoritmalarının dozimetrik doğruluğunu araştırmışlar. Fantomda yaptıkları IMRT ve IMAT planlarında TLD ölçümlerini AAA algoritması için \%0,2 ile \%4,6 arasında bulmuşlardır. Çalışmamızda AAA algoritması için TLD ölçümleriyle bulduğumuz fark IMRT ve IMAT planlarında \% 1.01 ile \% 3.5, hibrit planda ise \%1,04 ile \%3,16 arasındadir. 
Szu-Huai Lu ve ark. (16) 20 hasta üzerinde SIB tekniğiyle IMRT ve IMAT planlarını karşılaştırdıkları çalışmada MU değerlerini sırasıyla 949,3 ve 693,1 MU bulmuşlardır. Aynı çalışmada 1şınlama süreleri sırasıyla 9,5 dk ve 5,7 dk dır. Tedavi süresi ve MU bizim çalışmamızın sonuçları gibi IMAT planda daha azdır.

Günümüzde Nazofarenks planlaması için Hibrit tedaviler gündemdedir. Özellikle IMAT veya IMRT planlarında düşürülemeyen kritik organ dozları veya iyi kapsanamayan PTV hacimleri için Hibrit planlara ihtiyaç duyulmaktadır. Literatürde IMRT ve IMAT planlarının dozimetrik doğruluğu ile ilgili birçok çalışma var iken Hibrit planlama için bir çalışma bulunmamaktadır. Biz bu çalışma ile Nazofarenks için yapılan Hibrit tedavi planının TPS de doğruluğunun \%4 ten az olduğunu dozimetrik olarak tespit ettik.

\section{Teșekkür}

$\mathrm{Bu}$ çalışma, "Radyasyon Onkolojisinde Tümör Kontrol Oranlarının Artırılması ve Volümetrik Ark Tedavilerinin Radyasyon Onkolojisinde Yan Etkilerinin Azaltılması" isimli İstanbul Üniversitesi BAP projesi kapsamında güncellenmiş TPS (Eclipse 15.1) versiyonuyla gerçekleştirilmiştir (Proje ID: 23057).

\section{Kaynakça}

[1] M. Altun, A. Fandi, O. Dupuis, et al. "Undifferentiated nasopharyngeal cancer (UCNT): Current diagnostic and therapeutic aspects," Int J Radiation Oncology Biol Phys., 32(3), 859-877, 1995.

[2] C. P. Chang, T.F. Liu, Y.W. Chang, et al. "Radiation therapy of nasopharyngeal carcinoma," Acta Radiol Oncol., 19, 433-438, 1980.

[3] A.W.M. Lee, S.C.K. Law, and W. Foo, "Retrospective analysis of patients with nasopharyngeal carcinoma treated during 1976-1985: Overall survival and patterns of failure," Int J Radiat Oncol Biol Phys., 23, 261-270, 1992.

[4] G. Vincent et al.'Intensity modulated Radiation Therapy For Head and Neck Carcinama," The oncologist., 12, 555-564, 2007.

[5] S.A. Bhide et al. "The role of intensity-modulated radiotherapy in head and neck cancer," Indian J Cancer.; 47(3), 267-73. 2010

[6] Journal of the ICRU Report 83, "Prescribing, Recording and Reporting Photon-Beam IntensityModulated Radiation Therapy (IMRT) UK”, 10 (1), 2010.

[7] Contribution Of Teaching Intervention To Set-Up Verification With CBCT for Prostate Cancer, ESTRO 29, Radiotherapy \& Oncology Congress, Barcelona, pp. 1272, 2010.

[8] W. F. A. R. Verbakel, J. P. Cuijpers, D. Hoffmans, et al., "Volumetric intensity-modulated arc therapy vs. conventional IMRT in head-and-neck cancer: a Comparative Planning and Dosimetric Study," International Journal of Radiation Oncology Biology Physics., 74 (1), 252-259, 2009.

[9] E. Vanetti, A. Clivio, G. Nicolini et al., "Volumetric modulated arc radiotherapy for carcinomas of the oro-pharynx, hypopharynx and larynx: a treatment planning comparison with fixed field IMRT," Radiotherapy and Oncology., 92 (1), 111-117, 2009.

[10] T.-F. Lee, H.-M. Ting, P.-J. Chao, and F.-M. Fang, "Dual arc volumetric-modulated arc radiotherapy (VMAT) of nasopharyngeal carcinomas: a simultaneous integrated boost treatment plan comparison with intensity-modulated radiotherapies and single arc VMAT," Clinical Oncology., 24(3), 196-207, 2012.

[11] Z. H. Ning, J. M. Mu, J. X. Jin et al., "Single arc volumetricmodulated arc therapy is sufficient for nasopharyngeal carcinoma: a dosimetric comparison with dual arc VMAT and dynamic MLC and step-and-shoot intensity-modulated radiotherapy," Radiation Oncology., 8(1), article 237, 2013.

[12] Z. Nan, Y. Ruijie, J. Yuliang, et. al,“ A Hybrid IMRT/VMAT Technique for the Treatment of Nasopharyngeal Cancer,” Bio. Med. Research International., (2015), pp. 1-8, 2015.

[13] Y. Sun, R. Guo, W. J. Yin, L. L. Tang, et al., “ Which T Category of Nasopharyngeal Carcinoma May Benefit Most from Volumetric Modulated Arc Therapy Compared with Step and Shoot Intensity Modulated Radiation Therapy", PLoS ONE, 8(9), 1-8, 2013.

[14] E. C. Laurence, J. D. C. Lennart, S. Jun, et al., “ Dynamic IMRT Treatments of Sinus Region Tumors: Comparison of Monte Carlo Calculations with Treatment Planning System Calculations and Ion Chamber Measurements," Technology in Cancer Research and Treatment, 5(5), 489-95, 2006. 
[15] T. Han, D. Followill, J. Mikell, et. al., "Experimental validation of deterministic Acuros XB algorithm for IMRT and VMAT dose calculations with the Radiological Physics Center's head and neck phantom," Med. Phys., 39(4), 2193-2202, 2012.

[16] L. Szu-Huai, C. J. Chia-Hsien, S. Kuo, et al. "Volumetric modulated arc therapy for nasopharyngeal carcinoma: A dosimetric comparison with TomoTherapy and step-and- shoot IMRT," Radiotherapy and Oncology., 104, 324-330, 2012. 